\title{
The spectrum of eosinophilic infiltration of the cecum and its relationship to other disorders of multiple granulomas and arteritis in Sprague-Dawlay rat
}

\author{
Yinghua $\mathrm{Li}^{1}$, Han-lk Bae${ }^{2}$, Hak-Soo Kim¹, Min-Soo Kang ${ }^{1}$, Won-Hee Jung ${ }^{1}$, \\ Kap-Ho Kim ${ }^{1}$, Si-Whan Song ${ }^{1}$ and Boo-Hyon Kang ${ }^{1}$ \\ ${ }^{1}$ Department of Pathology, Chemon Co. Ltd., 240, Nampyeongro, Yangji-Myeon, Cheoin-Gu, Yongin-Si, \\ Gyeonggi-Do, 449-826, Republic of Korea \\ ${ }^{2}$ Department of Pathology, Kyungpook National University Medical Center, 474, Hakjeongdong, Buk-gu, Daegu, \\ 702-210, Republic of Korea
}

(Received November 5, 2013; Accepted January 7, 2014)

\begin{abstract}
Spontaneous multiple granulomas were present in the animal under the SPF condition and without chemical treatment, in a 19-week-old male Sprague-Dawley control-group rat. Here we describe multiple granulomas and prominent diffuse infiltration by eosinophils in the cecal submucosa, and arteritis in the mesenteric arteries. The multiple granulomas were characterized by central eosinophilic degeneration or necrosis, prominent eosinophils, many multi-nucleated giant cells and abundant fibroblasts. They were restricted to the cecal submucosa. The mesenteric arteritis consisted of fibrinoid necrosis of the intima and media, intense inflammatory cell infiltration and fibrosis in the arterial wall. An affected artery in the cecum was continuous with the mesenteric artery. The foregoing tissue changes in this rat correlate with the high absolute blood eosinophil count found in this animal.
\end{abstract}

Key words: Arteritis, Cecum, Eosinophilic infiltration, Multiple granulomas

\section{INTRODUCTION}

Eosinophils are leukocytes derived from the bone marrow. Eosinophils are increased in reactive diseases such as allergic and parasitic diseases, some malignant diseases, immunodeficiency diseases, and in idiopathic hypereosinophilic syndrome (HES) (Matsumoto et al., 2000; Gleich and Adolphson, 1986).

Eosinophils play major roles as mediators of tissue damage as well as wound healing. Of particular interest concerning the tissue-damaging potential of eosinophils has been the recent description of the intracellular contents of eosinophils. These include major basic protein, eosinophilic cationic protein, and eosinophilic peroxidase. Each of these proteins has been cloned, and their biochemical and functional properties have been well studied (Brito-Babapulle, 2003; Sano et al., 2001; Weller et al., 1988). Eosinophils become activated, and acquire increased cytotoxic capacity. The presence of activated cells and their secreted products in granulomas support the view that they are possible pathogenetic components in these lesions. Activated and degranulating eosinophils were also observed in the adjacent blood vessels and around the necrotic lesion. Eosinophilic granule proteins have a considerable capacity to damage tissues and cells (Tai et al., 1984).

In a 19 -week-old SD rat, we recently observed prominent eosinophil infiltration and multiple granulomas in the cecum and arteritis in the mesenteric artery. The purpose of this report is to describe a spectrum of lesions seen in an SD rat, in which eosinophil infiltration, multiple granulomas, and arteritis are associated with high blood eosinophil count.

\section{MATERIALS AND METHODS}

\section{Animal}

The animal was a male SPF Sprague Dawley (Hsd; SD) rat purchased from Koatech Inc., Republic of Korea. It was used as a vehicle control animal in a 13-week 
repeated-dose toxicity study and was submitted for sacrifice at the end of the study. The animal was housed in a solid-bottom polycarbonate cage under routine controlled conditions (temperature, $23 \pm 3^{\circ} \mathrm{C}$; humidity, $55 \% \pm$ $15 \%$; lighting, $12 \mathrm{hr}$ ) and had free access to Teklad-certified, irradiated, global $18 \%$ protein rodent diet $2918 \mathrm{C}$ (Harlan Laboratories, Inc., Hayward, CA, USA) and sterilized tap water.

This animal was housed in a facility (Chemon Co. Ltd., Yongin-si, Gyeonggi-do, Republic of Korea) approved by the Association for Assessment and Accreditation of Laboratory Animal Care International (AAALAC). The study was in compliance with Good Laboratory Practice (GLP) standards and followed the Test Guidelines of the Organization for Economic Cooperation and Development (OECD, 1998a, 1998b) and the Korea Food and Drug Administration (KFDA, 2009a, 2009b).

\section{Clinical signs}

The animal was observed daily for clinical signs and mortality. Clinical signs were recorded individually. The initial day of vehicle administration was set as Day 1.

\section{Body weight}

The body weight was recorded on the date of initial administration, weekly, and on the day of necropsy. The body weight on the necropsy date was measured after an overnight fast.

\section{Food and water consumption}

The food and water consumption were recorded weekly after treatment was begun, calculated as the difference between the supplied amounts and the remaining amounts measured on the following week.

\section{Ophthalmological examination}

In the last week of the experiment the eyes were examined through a fundus camera (Genesis, Kowa Co., Tokyo, Japan), after the pupil was dilated with a mydriatic (Ocuhomapin, Lot No. 022519, Samil Pharm Co., Seoul, Republic of Korea). The observation areas included the cornea, conjunctiva, sclera, iris, and fundus.

\section{Urinalysis}

Urine was collected for determination of routine urinalysis. Urine measurements (measured by an automatic analyzer: Clini-Tek 100, Siemens, Ames Division, Miles Laboratory, Tarrytown, NY, USA; Test strip: Multistix 10SG, Siemens, Washington, DC, USA) included the following parameters: specific gravity, $\mathrm{pH}$, protein, glucose, ketone bodies, occult blood, bilirubin, urobilinogen, clarity, nitrite, and urine color. The sediment was stained using the Sternheimer-Malbin method (Sternheimer and Malbin, 1951). Red blood cells (RBCs), white blood cells (WBCs), epithelial cells, and casts were observed under a microscope.

\section{Hematology and serum biochemistry}

Blood for routine hematological and serum biochemical analysis was obtained under deep anesthesia, before the rat was sacrificed by exsanguination from the posterior vena cava.

Hematological measurements (measured by ADVIA 2120, Siemens, Ames Division, Miles Laboratory, Tarrytown, NY, USA; ACL 100, Instrumentation Laboratory, Bedford, MA, USA) included the following parameters: RBCs, hemoglobin concentration, hematocrit, mean corpuscular volume, mean corpuscular hemoglobin, mean corpuscular hemoglobin concentration, red cell distribution width, hemoglobin distribution width, mean platelet volume, reticulocytes, platelet count, WBCs, and WBC differential count (neutrophils, lymphocytes, monocytes, eosinophils, basophils, and large unstained cells), prothrombin time, and activated partial thromboplastin time. The absolute numbers of lymphocytes and eosinophils (cells/ $\mu$ l) were calculated.

Serum biochemical analysis (measured by AU680, Olympus, Tokyo, Japan) included the following parameters: aspartate aminotransferase, alanine aminotransferase, alkaline phosphatase, creatine phosphokinase, total bilirubin, glucose, total cholesterol, triglycerides, total protein, albumin, albumin/globulin ratio, blood urea nitrogen, creatinine, inorganic phosphorus, calcium, sodium, potassium, and chloride.

\section{Necropsy and organ weights}

Before the scheduled necropsy date, the rats were fasted overnight (for 16-20 hr) and anesthetized with isoflurane inhalation (Ifran liquid, Hana Pharm. Co., Seoul, Republic of Korea) on the day of necropsy. After anesthesia was confirmed, blood samples were taken from the posterior vena cava for hematology and serum biochemistry, and the animals were sacrificed by exsanguination. The external surfaces, subcutis, all orifices and all organs in the cranial cavity, abdominal and thoracic cavities, and their contents were observed grossly. Next, the pituitary gland, ovary, uterus, adrenal gland, thymus, prostate gland, testes, epididymis, spleen, kidney, heart, lung, brain, and liver were removed and weighed with an electronic balance (BP221S, Sartorious Co., Gottingen, Lower Saxony, Germany), and all paired organs were measured separately. The absolute organ weights were converted to relative 
The spectrum of eosinophilic infiltration and relationship to multiple granulomas and arteritis

organ weights based on the organ-to-fasted body weight ratio.

\section{Histopathology}

The eyes with the optic nerve were preserved in Davidson's fixative, and the testes and epididymis were preserved in Bouin's fixative. The following organs and tissues were fixed in 10\% neutral buffered formalin solution: thymus, spleen, pancreas, stomach, duodenum, jejunum, ileum, cecum, colon, rectum, mesenteric lymph node, mesenteric artery, mandibular lymph node, salivary gland, thyroid gland (including parathyroid gland), Harderian gland, heart, lung, kidney, adrenal gland, liver, aorta, brain, pituitary gland, tongue, trachea, esophagus, thoracic spinal cord, bone marrow in sternum and femur, femorotibial joint, peripheral nerve (sciatic), skeletal muscle (femoral), prostate gland, seminal vesicle, ovary, uterus, vagina, urinary bladder, and skin (including mammary gland). The fixed specimens were embedded in paraffin, sectioned at 4- $\mu$ m thickness, stained with hematoxylin and eosin (H\&E), and examined microscopically.

In addition, in this rat, the cecum and mesenteric artery were stained using Gram and acid-fast stains to reveal bacteria. Giemsa and periodic acid-Schiff (PAS) were used to stain fungi or parasitic remnants. Masson's trichrome staining was used to stain fibrotic tissue. To detect eosinophils as described previously (Grouls and Helpap, 1981), the sections were also stained with Congo red and were finally counterstained with hematoxylin. Verhoeff's elastic fiber staining was also used to show the elastic tissues and evaluate the degree of destruction of the artery and arteriole.

\section{RESULTS}

\section{Clinical signs and laboratory findings}

There were no remarkable changes in clinical signs, body weight, food and water consumption, ophthalmological examination, urinalysis, serum biochemistry, and organ weights in this case (data not shown).

In hematological parameters, the calculated absolute eosinophil count was 107 cells/ $\mu 1$ in this case. In the other nine male rats of this control group, the mean eosinophil count was $75 \pm 23$ cells/ $\mu 1$ in this study. There were no remarkable changes in other hematological parameters (data not shown).

\section{Necropsy and histopathological findings}

At necropsy, thickening of the wall was observed in the cecum, and there were no remarkable changes in other organs.
Histopathologically, large numbers of eosinophils were prominent in the lamina propria, submucosa, and muscularis of the cecum (Fig. 1). Fibrosis was accompanied by infiltration of numerous eosinophils that was confirmed by Masson's trichrome stain (Fig. 2A) and, occasionally, deposition of granular eosinophilic material associated with macrophages (Fig. 1C). Additionally, multiple granulomas were found in the cecal submucosa (Fig. 1) and Elastic fibers in a granulomatous area of the cecal submucosa were confirmed by Verhoeff's elastic fiber stain (Fig. 2B). They showed central, degenerated or necrotic eosinophilic cores surrounded by epithelioid macrophages and multi-nucleated giant cells (Figs. 1B and 1C). Such structures sometimes resembled epithelioid tubercles. Eosinophil infiltration often disrupted the normal architecture, resulting in atrophy of the cecal glands (Fig. 1A).

In the mesenteric artery, mild to moderate segmental, sometimes necrotizing, arteritis with mural thrombosis and transmural infiltration of eosinophils were present. The lumen was markedly narrowed by the thickened edematous intima and at times occluded by thrombi (Fig. 3). Furthermore, the affected artery in the cecum was continuous with the mesenteric artery which had similar changes. The prominent eosinophil infiltration was found in all layers of the arterial wall (Fig. 4).

Slight increase of extra-medullary leukopoesis in the spleen and slight increase of eosinophils in the bone marrow were also observed, all of these change to supply eosinophils to the cecum and affected arteries.

Microorganisms were not observed in Gram, acid-fast, Giemsa, and PAS-stained sections. Giemsa stain did not reveal mast cells.

\section{DISCUSSION}

An accumulation of eosinophils occurs in several important disorders, such as allergic and parasitic disease, some malignant diseases, immunodeficiency diseases, and in idiopathic HES (Matsumoto et al., 2000; Rothenberg, 1998; Weller, 1991). In certain disease states, eosinophils can selectively accumulate in peripheral blood or any tissue in the body.

Matsumoto et al. (2000) reported that the mean normal count of blood eosinophils is 80 cells $/ \mu 1$ in rats. In the rat in this case, the eosinophil count (107 cells/ $\mu$ l) was higher than the mean value of the other nine male rats of its control cohort, and also higher than the number reported by Matsumoto. Therefore, the tendency of increased eosinophils was observed in this rat.

Histopathologically, the diffuse form of eosinophilic infiltration and multiple granulomas in the cecum in this 


\section{Y. Li et al.}
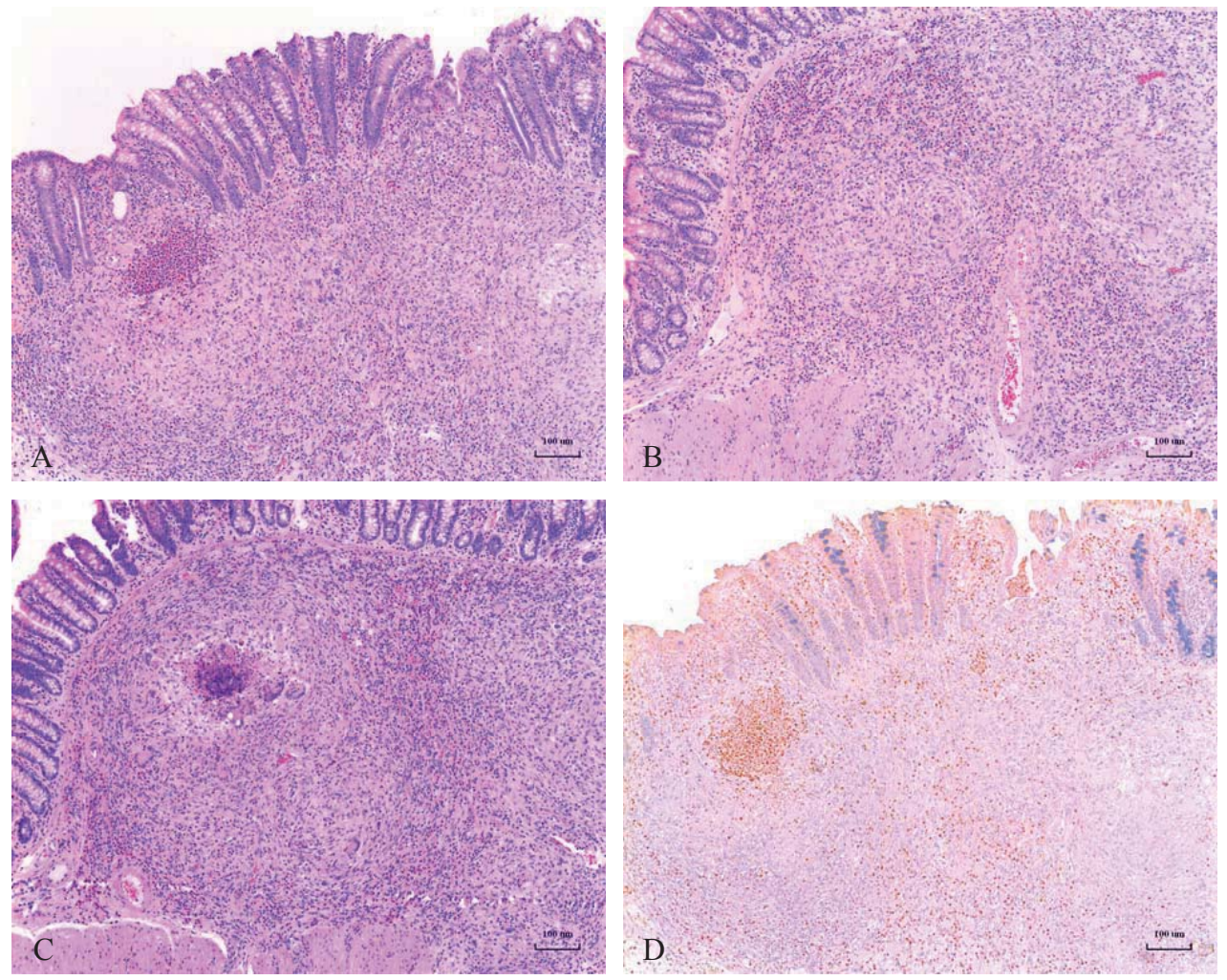

Fig. 1. Histopathological features of the cecum. A: The eosinophilic infiltration often disrupts the normal architecture, associated with atrophy of the cecal glands. H\&E stain. Scale bars $=100 \mu \mathrm{m}$. B: The numerous eosinophils are present. H\&E stain. Scale bars $=100 \mu \mathrm{m}$. C: Multiple granulomas were present in the cecal submucosa. They had degenerated or necrotic eosinophilic centers surrounded by epithelioid macrophages, multi-nucleated giant cells, and prominent eosinophil infiltration. H\&E stain. Scale bars $=100 \mu \mathrm{m}$. D: The prominent eosinophilic infiltration in the cecum was confirmed by Congo red stain. Scale bars $=100 \mu \mathrm{m}$.
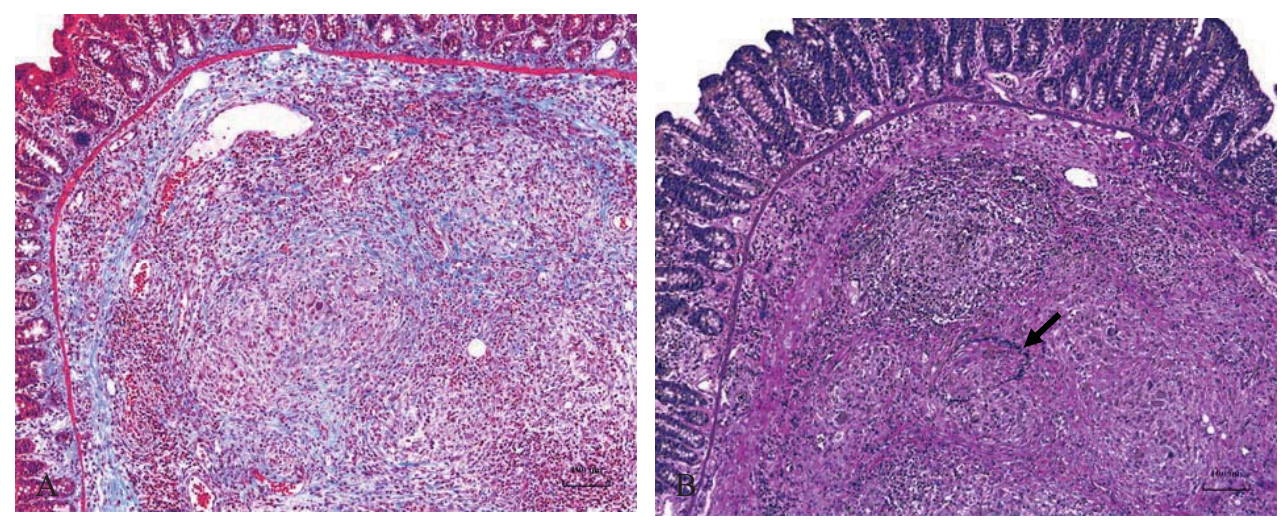

Fig. 2. A: Prominent fibrosis was accompanied by infiltration of numerous eosinophils into the cecum, here confirmed by Masson's trichrome stain. Scale bar $=100 \mu \mathrm{m}$. B: Elastic fibers (arrow) in a granulomatous area of the cecal submucosa were confirmed by Verhoeff's elastic fiber stain. Scale bar $=100 \mu \mathrm{m}$. 
The spectrum of eosinophilic infiltration and relationship to multiple granulomas and arteritis
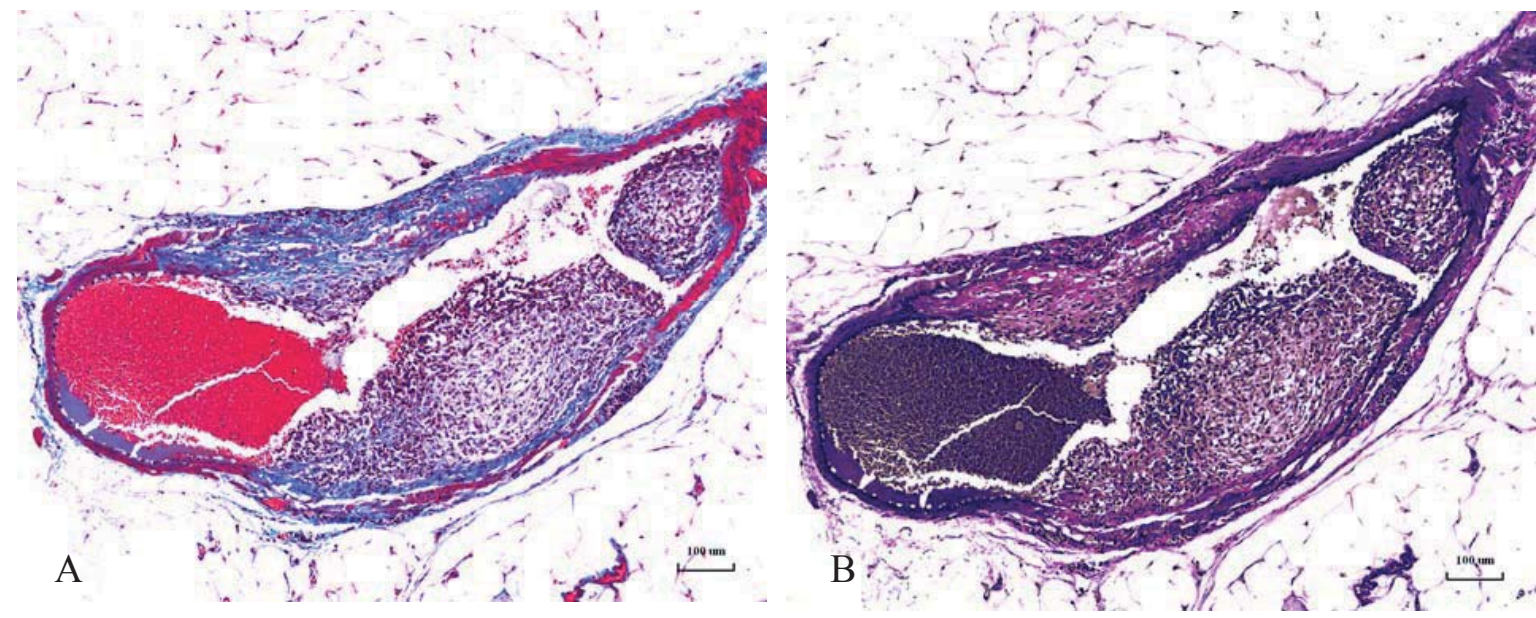

Fig. 3. Histopathological features of the mesenteric artery. A: The mesenteric artery had intimal fribrinoid necrosis and proliferation, with luminal narrowing by thrombosis. Masson's trichrome stain. Scale bar $=100 \mu \mathrm{m}$. B: The elastic fibers were disrupted in affected mesenteric artery, confirmed here by Verhoeff's elastic fiber stain. Scale bar $=100 \mu \mathrm{m}$.

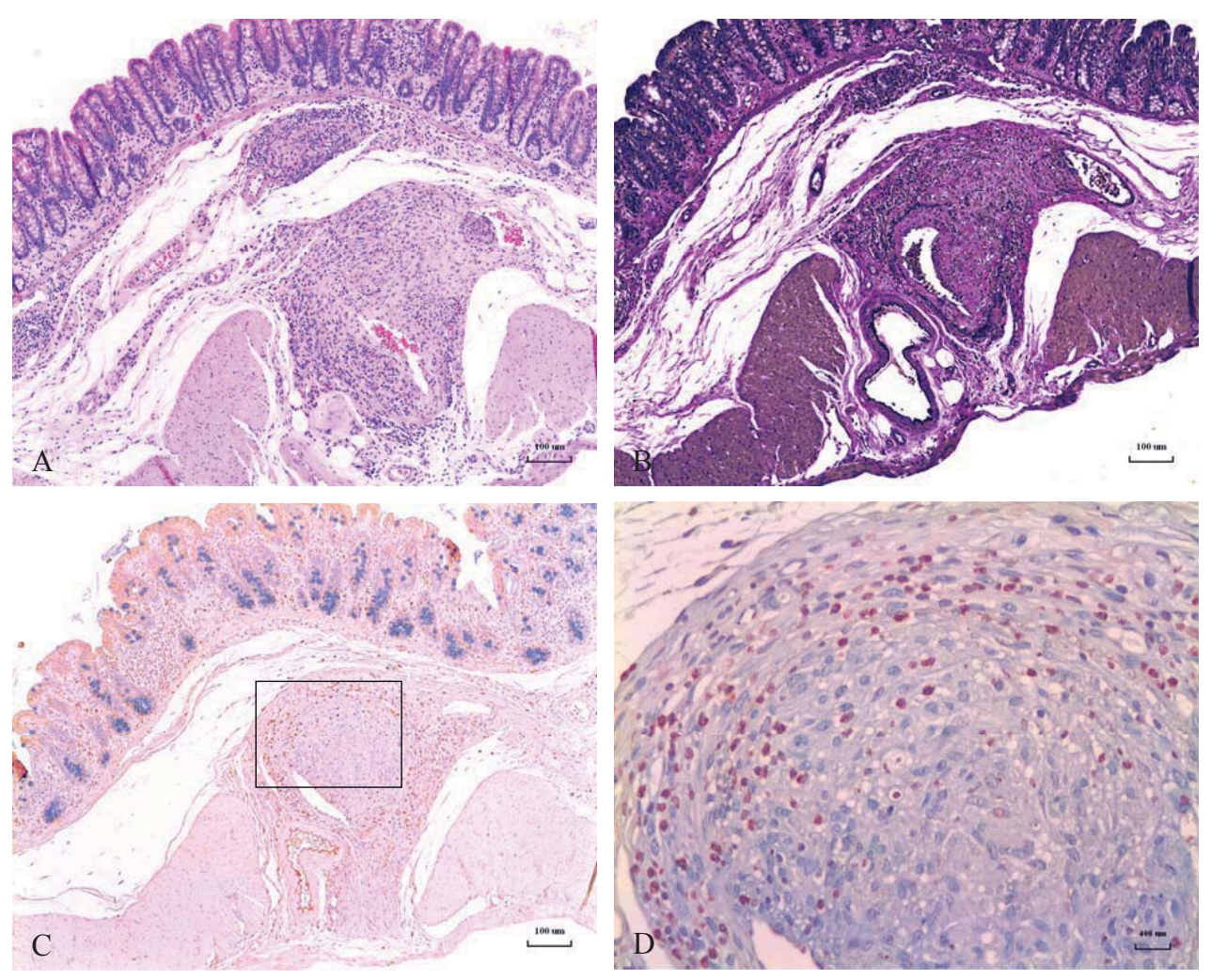

Fig. 4. A: An affected artery in the cecum which was continuous with the affected mesenteric artery (Fig. 3) contained similar changes. H\&E stain. Scale bar $=100 \mu \mathrm{m}$. B: Disruption of elastic fibers was confirmed by Verhoeff's elastic fiber stain. Scale bar $=$ $100 \mu \mathrm{m}$. C: Transmural infiltration of eosinophils was confirmed by Congo red stain. Scale bar $=100 \mu \mathrm{m}$. D: High magnification of Fig. 4C. Eosinophils infiltrated all layers of affected arterial walls. Congo red stain. Scale bar $=400 \mu \mathrm{m}$. 
case appeared to be similar to that described in other species (Fox et al., 1992; Hendrick, 1981; Pass and Bolton, 1982). This was characterized by prominent infiltration by eosinophils with resultant thickening of the affected portion of the cecum. Tissue eosinophilia and its degranulation in situ have been suggested to be a major contribution to development of tissue injury (Hällgren et al., 1991; Sano et al., 2001).

A number of studies have reported the ability of various eosinophilic components to cause tissue damage (Grantham et al., 1986; Leiferman et al., 1985; Sano et al., 2001; Tai et al., 1987); however, relatively little attention has been given to tissue reaction within the lesions in SD rats. Eosinophilic abscesses with granulomatous inflammation have been reported. (Kanner and Hammar, 1977; Sasano et al., 1989)

A characteristic feature of the granulomas in this rat was the macrophages and multi-nucleated giant cells containing phagocytosed eosinophilic material, surrounded by intense eosinophilic cell infiltration. In the literature illustrating HES, similar lesions were described (Sano et al., 2001; Sasano et al., 1989).

Tissue injury in the cecum in this case was considered to be caused by the release of the proteins from the distinctive cytoplasmic granules of the eosinophils. Many activated and degranulating eosinophils seemed to migrate from the blood into these areas.

In this case, arteritis was observed in the mesenteric artery and in its extension into the cecum was continuous. The damage in the mesenteric artery was characterized by infiltration of activated eosinophils into all layers of the inflamed vessel, suggesting that eosinophils were involved in pathogenesis in the lesions in the mesenteric arteries as well as in the cecum. Eosinophil granule proteins may contribute to the necrotic lesions in the artery wall and the development of thrombi.

A possible mechanism by which eosinophil granule proteins induce cell damage has been elucidated by Young et al. (1986). The cytotoxic properties of the eosinophil cationic protein and major basic protein suggested that their extracellular deposition may contribute to vascular injury (Hällgren et al., 1991; Young et al., 1986). These eosinophil constituents have been demonstrated to be harmful to endothelial cells in vitro, thus suggesting they are etiological agents of vasculitis (Brito-Babapulle, 2003; Hällgren et al., 1991) or at least, contribute to the pathogenesis of the lesion.

In present study, this rat showed tendency of increased eosinphils complicated with eosinophil-related lesions. What causes increased eosionphils in this rat? Specific infection leading to an increase in eosinophils was doubt- ful because no evidence of larval or adult parasites and no significant microorganisms were found histopathologically. Furthermore, this animal was maintained under SPF conditions and was also free from Aspergillus funmigatus infection which is known to cause eosinophilia (Matsumoto et al., 2000). Although we could not reveal mast cells with Giemsa stain, we were unable to completely rule out an allergic etiology, because we were unable to evaluate serum immunoglobulin E concentration. There was no histopathologic evidence of leukemia or other neoplasm as a cause for this eosinophilia. Therefore, details of pathology and pathogenesis of increased eosinophils in this case were unclear.

Tissue deposition of eosinophils and release of eosinophilic granule products were pathophysiological cause of tissue and organ damage. Although eosinophilic constituents largely contribute to the development of vasculitis or tissue injury, what triggers eosinophilic infiltration to the tissues and degranulation of eosinophil in situ remains to be clarified.

In conclusion, we describe eosinophil infiltration, multiple granulomas, and arteritis in an SD rat in the vehicle control group and we discuss pathogenesis of tissue injury related to a prominent infiltration of eosinophils count in this case. However, the specific pathogenesis of these disorders is not known and further studies are required to determine the actual relationships among cause of increased eosinophils and the organ-targeting inflammation.

\section{ACKNOWLEDGMENTS}

The authors would like to thank technicians at the Department of Pathology of Chemon Co. Ltd. for technical assistance.

\section{REFERENCES}

Brito-Babapulle, F. (2003): The eosinophilias, including the idiopathic hypereosinophilic syndrome. Br. J. Haematol., 121, 203223.

Fox, J.G., Palley, L.S. and Rose, R. (1992): Eosinophilic gastroenteritis with Splendore-Hoeppli material in the ferret (Mustela putorius furo). Vet. Pathol., 29, 21-26.

Gleich, G.J. and Adolphson, C.R. (1986): The eosinophilic leukocyte: structure and function. Adv. Immunol., 39, 177-253.

Grantham, J.G., Meadows, J.A. and Gleich, G.J. (1986): Chronic eosinophilic pneumonia. Evidence for eosinophil degranulation and release of major basic protein. Am. J. Med., 80, 89-94.

Grouls, V. and Helpap, B. (1981): Selective staining of eosinophils and their immature precursors in tissue sections and autoradiographs with Congo red. Stain. Technol., 56, 323-325.

Hällgren, R., Gudbjörnsson, B., Larsson, E. and Fredens, K. (1991): Deposition of eosinophil cationic protein in vascular lesions in 
The spectrum of eosinophilic infiltration and relationship to multiple granulomas and arteritis

temporal arteritis. Ann. Rheum. Dis., 50, 946-949.

Hendrick, M. (1981): A spectrum of hypereosinophilic syndromes exemplified by six cats with eosinophilic enteritis. Vet. Pathol., 18, 188-200.

Kanner, R.E. and Hammar, S.P. (1977): Chronic eosinophilic pneumonia. Ultrastructural evidence of marked immunoglobulin production plus macrophagic ingestion of eosinophils and eosinophilic lysosomes leading to intracytoplasmic Charcot-Leyden crystals. Chest, 71, 95-98.

KFDA (2009a): Good Laboratory Practice Regulation for Non-Clinical Laboratory Studies (Notification No. 2009-102). KFDA, Korea.

KFDA (2009b): The Standards of Toxicity Study for Medicinal Products (Notification No. 2009-116). KFDA, Korea.

Leiferman, K.M., Ackerman, S.J., Sampson, H.A., Haugen, H.S., Venencie, P.Y. and Gleich, G.J. (1985): Dermal deposition of eosinophil-granule major basic protein in atopic dermatitis. Comparison with onchocerciasis. N. Engl. J. Med., 313, 282285.

Matsumoto, K., Matsushita, N., Tomozawa, H. and Tagawa, Y. (2000): Hematological characteristics of rats spontaneously developing eosinophilia. Exp. Anim., 49, 211-215.

OECD (1998a): OECD Principles of Good Laboratory Practice ENV/MC/CHEM (98) 17. OECD, Paris.

OECD (1998b): Guidelines for Testing of Chemicals. Guideline 408. Repeated Dose 90-day Oral Toxicity Study in Rodents. OECD, Paris.

Pass, D.A. and Bolton, J.R. (1982): Chronic eosinophilic gastroenteritis in the horse. Vet. Pathol., 19, 486-496.
Rothenberg, M.E. (1998): Eosinophilia. N. Engl. J. Med., 338, $1592-1600$

Sano, K., Kobayashi, M., Sakaguchi, N., Ito, M., Hotchi, M. and Matsumoto, K. (2001): A rat model of hypereosinophilic syndrome. Pathol. Int., 51, 82-88.

Sasano, H., Virmani, R., Patterson, R.H., Robinowitz, M. and Guccion, J.G. (1989): Eosinophilic products lead to myocardial damage. Hum. Pathol., 20, 850-857.

Sternheimer, R. and Malbin, B. (1951): Clinical recognition of pyelonephritis, with a new stain for urinary sediments. Am. J. Med., 11, 312-323.

Tai, P.C., Ackerman, S.J., Spry, C.J., Dunnette, S., Olsen, E.G. and Gleich, G.J. (1987): Deposits of eosinophil granule proteins in cardiac tissues of patients with eosinophilic endomyocardial disease. Lancet, 1, 643-647.

Tai, P.C., Holt, M.E., Denny, P., Gibbs, A.R., Williams, B.D. and Spry, C.J. (1984): Deposition of eosinophil cationic protein in granulomas in allergic granulomatosis and vasculitis: the ChurgStrauss syndrome. Br. Med. J. (Clin. Res. Ed.)., 289, 400-402.

Weller, P.F. (1991): The immunobiology of eosinophils. N. Engl. J. Med., 324, 1110-1118.

Weller, P.F., Ackerman, S.J. and Smith, J.A. (1988): Eosinophil granule cationic proteins: major basic protein is distinct from the smaller subunit of eosinophil peroxidase. J. Leukoc. Biol., 43, $1-4$.

Young, J.D., Peterson, C.G., Venge, P. and Cohn, Z.A. (1986): Mechanism of membrane damage mediated by human eosinophil cationic protein. Nature, 321, 613-616. 